\title{
BGISEQ-500 Sequencing
}

National Cancer Institute

\section{Source}

National Cancer Institute. BGISEQ-500 Sequencing. NCI Thesaurus. Code C146812.

A proprietary next-generation DNA sequencing system from BGI Genomics using combinatorial Probe-Anchor Synthesis (CPAS) and improved DNA Nanoballs (DNB) technology. The cPAS chemistry works by incorporating a fluorescent probe to a DNA anchor on the DNB, followed by high-resolution digital imaging. 